\title{
Tác động của trách nhiệm xã hội của doanh nghiệp lên chất lượng mối quan hệ thương hiệu - khách hàng và ý định chuyển đổi thương hiệu tại Việt Nam
}

\section{The impact of corporate social responsibility on brand - customer relationship quality and brand switching intention in Viet Nam}

\author{
Trần Nguyễn Khánh Hải ${ }^{*}$, Nguyễn Quang Thu ${ }^{2}$ \\ ${ }^{1}$ Trường Đại học Tài chính - Marketing, Việt Nam \\ ${ }^{2}$ Trường Đại học Kinh tế Thành phố Hồ Chí Minh, Việt Nam \\ *Tác giả liên hệ, Email: tnkhanhhai@gmail.com
}

THÔNG TIN

DOI: $10.46223 /$ HCMCOUJS.

econ.vi.13.2.516.2018

Ngày nhận: 26/02/2018

Ngày nhận lại: 05/04/2018

Duyệt đăng: 09/04/2018

Tù khóa:

chất lượng mối quan hệ thương hiệu - khách hàng, trách nhiệm xã hội của doanh nghiệp, ý định chuyển đổi thương hiệu

Keywords:

brand-customer relationship quality, corporate social responsibility, brand switching intention
TÓM TẮT

Nghiên cứu này nhằm kiểm định và đánh giá tác động của trách nhiệm xã h ội củ a doanh nghiệp (CSR) lên chất lượng mối quan hệ thương hiệu - khách hàng và ý định chuyển đổ i thương hiệu. Mối quan hệ này được kiểm chứng thông qua phân tích 507 mẫu khách hàng thường xuyên mua sắm tại các siêu thị bán lẻ. Kết quả cho thấy, CSR có tác động mạnh mẽ, cùng chiều lên chất lượng mối quan hệ thương hiệu - khách hàng; đồng thời CSR làm giảm ý định chuyển đổi thương hiệu của khách hàng. Nghiên cứu này cũng đề cập vai trò trung gian củ a chất lượng mối quan $\mathrm{h}$ ệ thương hiệu - khách hàng trong mối quan hệ giữa CSR và ý định chuyển đổi thương hiệu. Cuối cùng, nghiên cứu đưa ra kết luận và hàm ý chính sách cho các doanh nghiệp và hướng nghiên cứu tiếp theo.

\section{ABSTRACT}

This study aims to determine and assess the impact of Corporate Social Responsibility (CRS) on brand-customer relationship quality and brand switching intention. These relationships were determined by analyzing a sample of 507 regular customers shopping at retail supermarkets. The findings showed that CSR has a strong impact on the quality of the brandcustomer relationship while reduces brand switching intention. This study also discussed the mediating role of brand-customer relationship quality in the CSR-brand switching intention relationship. Finally, the study drew some conclusions and made some policy implications for enterprises and suggestions for future research. 


\section{Giới thiệu}

Nuôi dưỡng mối quan hệ giữa khách hàng và thương hiệu có chất lượng cao là điều quan trọng hàng đầu, đặc biệt đối với các doanh nghiệp bán lẻ vì trong lĩnh vực dịch vụ, khách hàng thường phải đối mặt với sự không chắc chắn, dẫn đến nhiều khả năng thất bại về dịch vụ và những kết quả tiêu cực (Crosby, Evans, \& Cowles, 1990). Thành công trong việc xây dựng mối quan hệ với khách hàng sẽ giảm thiểu được số khách hàng chuyển đổi thương hiệu.

Với xu hướng mở cửa thị trường theo các cam kết hiệp định thương mại tự do song phươ ng, đa phương cùng với sự tham gia ngày càng nhiều của các nhà bán lẻ lớ $n$ trên thế giới tại Việt Nam và sự mở rộng mạng lưới, đa dạng mô hình kinh doanh cũng như thâu tóm hệ thống kinh doanh của các nhà bán lẻ của các nhà đầu tư nước ngoài đã và đang tạo ra những cơ hội, thách thức lớn đối với doanh nghiệp trong nước.

Thêm vào đó, trình độ dân trí ngày càng cao; chất lượng của hàng hóa, dịch vụ ngày càng tốt; các chương trình marketing ngày càng hấp dẫn thì để tồn tại và thành công, đòi hỏi các thương hiệu siêu thị cần phải tăng cường mối quan hệ với khách hàng để nâng cao cạnh tranh. Vì vậy, việc nghiên cứu cách thiết lập mối quan hệ mật thiết với khách hàng là hết sức quan trọng đối với các thương hiệu siêu thị tại Việt Nam. Về mặt này, trách nhiệm xã hội thực hiện khá tốt, nó liên quan đến việc xây dựng mối quan hệ bằng cách quan tâm đến những lợi ích của họ. Thông qua trách nhiệm xã hội của doanh nghiệ p có thể khiến khách hàng hài lòng và doanh nghiệp có thể hưởng lợi từ mối quan hệ mật thiết này.

Nhận thức về CSR góp phần tăng cường lợi thế cạnh tranh của doanh nghiệp thông qua việc tăng cường mối quan hệ với khách hàng (Carroll \& Shabana, 2010; Lacey, Kennett-Hensel, \& Manolis, 2015; Su, Swanson, \& Chen, 2015b). Kết quả thực nghiệm của một số nghiên cứu đã cho thấy mối quan hệ vớ i khách hàng là một trung gian quan trọng của mối quan hệ kết quả CSR, cụ thể là sự hài lòng của khách hàng (Hur, Kim, \& Jang, 2016; Lev, Petrovits, \& Radhakrishnan, 2010); lòng tin của khách hàng (Hur et al., 2016; Vlachos, Tsamakos, Vrechopoulus, \& Avramidis, 2009); lòng trung thành của khách hàng (Nyadzayo \& Khajehzadeh, 2016). Tuy nhiên vẫn chưa có nghiên cứu nào đánh giá tác động của CSR lên ý đị nh chuyển đổi thương hiệu thông qua trung gian chất lượng mối quan hệ thương hiệu - khách hàng.

Mục tiêu của nghiên cứu này là xem xét vai trò trung gian của yếu tố chất lượng mối quan hệ thương hiệu - khách hàng trong mối quan hệ giữa CSR và ý đị nh chuyển đổi thương hiệu, với hai đóng góp mới:

- Kiểm định tác động trực tiếp từ CSR đến ý định chuyển đổi thương hiệu;

- Đánh giá vai trò trung gian của chất lượng mối quan hệ thương hiệu - khách hàng trong mối quan hệ giữa CSR và ý định chuyển đổi thương hiệu.

Các phần tiếp theo của bài viết này bao gồm: 1) Cơ sở lý thuyết và lược khảo các công trình nghiên cứu liên quan; (2) phương pháp nghiên cứu; (3) Kết quả và thảo luận; (4) Kết luận và hàm ý chính sách. 


\section{Cơ sở lý thuyết và các nghiên cứu liên quan}

\section{Lý thuyết bộ ba cốt lõi bền vũng (Triple-Bottom Line theory - TBL)}

Lý thuyết này đề cập lần đầu tiên trong nghiên cứu của Elkington và cộng sự vào năm 1995. TBL là một khung phân tích về kế toán để đo lường và lập báo cáo về hoạt động của doanh nghiệp về cả ba phương diện là kinh tế, xã hội và môi trường để khuyến khích các tổ chức kêt hợp tính bền vững vào thực tiễn kinh doanh của họ (Elkington, 1997; Chow, 2011). Nhiều nhà nghiên cứu cho rằng doanh nghiệp bền vững là một trong những lợi ích kinh tế, xã hội và môi trường (Alvarado, Bigne, Aldas-Manzano, \& Curras-Perez, 2017; Öberseder, Schlegelmilch, Murphy, \& Gruber, 2014). Mô hình của lý thuyết bộ ba cốt lõi bền vững được thể hiện trong Hình 1.

Một số nghiên cứu dựa trên lý thuyết của phát triển bền vững cho thấy người tiêu dùng có thể phân biệt ba thành phần của cấu trúc CSR (Alvarado et al., 2017; Kale \& Singh, 2007), do đó, việc sử dụng lý thuyết này là hoàn toàn hợp lý.

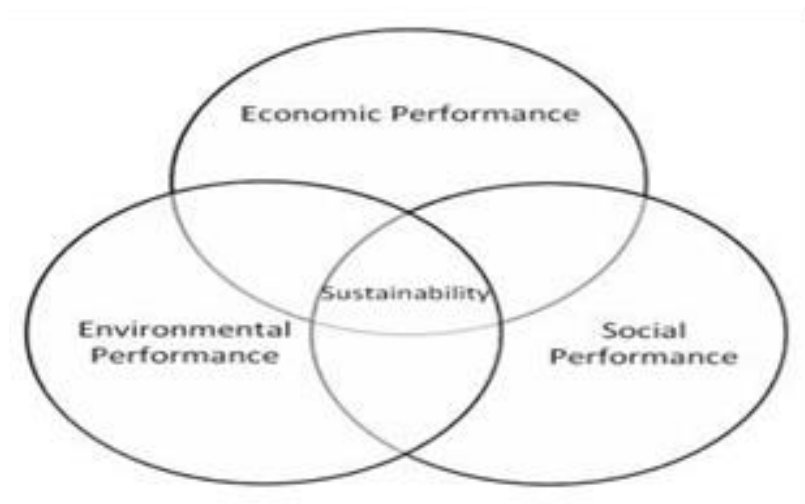

Hình 1. Mô hình lý thuyết bộ ba phát triển bền vừng -Triple Bottom Line

Nguồn: Elkington (1997)

Phát hiện này đặc biệt đúng trong trường hợp siêu thị bán lẻ tổng hợp (lĩnh vực được lựa chọn làm bối cảnh nghiên cứu) vì nó có các ưu điểm: siêu thị và các nhà cung cấp các sản phẩm cho siêu thị đang hoạt động trong các hoạt động CSR. Như vậy, lý thuyết TBL tạo ra cơ sở lý luận căn bản cho sự phát triển của CSR.

\subsection{Luọc khảo các công trình nghiên cứu thục nghiệm có liên quan}

Như đã giới thiệu ở trên, CSR "góp phần tăng cuờng lợi thế cạnh tranh của doanh nghiệp thông qua việc tăng cuờng mối quan hệ với khách hàng" (Carroll \& Shabana, 2010, p. 98). Đồng quan điểm này, Lacey và cộng sự (2015) cũng cho rằng nhận thức về CSR tăng cường chất lượng mối quan hệ với khách hàng của công ty đó. Nhiều nghiên cứu đã kiểm tra tác động của nhận thức CSR đến các kết quả marketing như: lòng trung thành của khách hàng (Fatma, Khan, \& Rahman, 2016; Lin \& Liu, 2017); từ đó, khách hàng đánh giá tích cực hơn về thương hiệu (Bhattacharya, Rao, \& Glyn, 1995).

CSR có ảnh hưởng trực tiếp và gián tiếp đến hành vi của khách hàng (Abdeen, Rajah, \& Gaur, 2016; Öberseder, Schlegelmilch, \& Gruber, 2011). Các nghiên cứu trước đây đã cho 
thấy những kết quả không nhất quán trong việc kiểm tra mối quan hệ giữa CSR và các hành vi của khách hàng (Loureiro, Sardinha, \& Reijnders, 2012; Pérez \& del Bosque, 2015). Điều này có thể kết luận là hiệu quả của CSR đối với hành vi của khách hàng sẽ phụ thuộc vào các biến trung gian và các tình huống thực tế (Carroll \& Shabana, 2010; Luo \& Bhattacharya, 2006).

Cho đến nay, chưa có nghiên cứu thực nghiệm nào được tiến hành để kiểm tra vai trò của CSR trong việc nâng cao chất lượng mối quan hệ giữa khách hàng và thương hiệu. Sự thiếu sót khi xem xét chất lượng của mối quan hệ thương hiệu - khách hàng đã được công nhận là "trung tâm trong xây dựng lý thuyết về tiếp thị mối quan hệ" (Smith \& Bolton, 1998, p. 4). Nhận thấy đây là một vấn đề khá mới và quan trọng nên nhóm đã chọn để nghiên cứu.

\subsection{Các khái niệm trong mô hình nghiên cúu và các giả thuyết}

\section{Trách nhiệm xã hội của doanh nghiệp}

Theo Du, Bhattacharya, và Sen (2011, p. 1) thì "trách nhiệm xã hội của doanh nghiệp là cam kết của một công ty để tối đa hóa kinh tế, xã hội và môi trương lâu dài thông qua các hoạt động kinh doanh, các chính sách và các nguồn lực". Định nghĩa này phù hợp với nghiên cứu vì nó cho phép xác định các thành phần CSR trực tiếp từ mô hình phát triển bền vững và tính hợp lý bền vững của nó nhằm mục đích quản lý và điều hành (Kakabadse \& Rozuel, 2006). Có thể hiểu, CSR là một cấu trúc đa chiều có ba đặc điểm - phát triển kinh tế, công bằng xã hội và bảo vệ môi trường - tương ứng với thành phần của phát triển bền vững (Van Marrewijk, 2003).

Trách nhiệm kinh tế: Sheth, Sethia, và Srinivas (2011) cho rằng thành phầ $\mathrm{n}$ kinh tế về tính bền vững gồm 2 vấn đề: (1) hiệu quả tài chính thông thường (e.g., giảm chi phí), và (2) lợi ích kinh tế của các bên liên quan bên ngoài (e.g., cải thiện kinh tế và tiêu chuẩn sống). Đối với người tiêu dùng, trách nhiệm kinh tế của doanh nghiệp là cung cấp hàng hóa và dịch vụ, định giá, thông tin về sản phẩm, phân phối, bán hàng và cạnh tranh.

Trách nhiệm xã hội: Trách nhiệm xã hội của một công ty là sự đáp ứng được tiêu chuẩn của một xã hội và sự sẵn lòng ủng hộ để làm cho nó trở thành một xã hội nhân đạo. Ngoài ra, quản lý hiệu quả và công bằng của một công ty đóng góp vào sự phát triển cho xã hội và nền kinh tế quốc gia nói chung (Fulop, Hisrich, \& Szegedi, 2000). CSR thúc đẩy doanh nghiệp phải chịu trách nhiệm với xã hội theo các khía cạnh khác nhau như nhân quyền, quyền bình đẳng và trả lương cho phụ nữ, đối xử bình đẳng trong một xã hội và bình đẳng với cạnh tranh với các đối tác.

Trách nhiệm môi trương: trách nhiệm môi trường đề cập đến nỗ lực để quả n lý các hoạt động cung cấp những sản phẩm, dịch vụ ít gây hại đến môi trường. Điều này được thể hiện trong các biện pháp ngăn ngừa ô nhiễm nước, đất và không khí. Cốt lõi của trách nhiệm môi trường đối vớ i một tổ chức là hoạt động trong phạm vi khả năng chịu đựng của hệ sinh thái bằng cách giả $\mathrm{m}$ ô nhiễm môi trường và gi ảm thiểu tiêu thụ tài nguyên (Lindgreen, Swaen, \& Johnston, 2009). 


\section{Chất lượng mối quan hệ thương hiệu - khách hàng}

Chất lượng mối quan hệ thương hiệu được định nghĩa là chỉ số dựa trên khách hàng về sức mạnh và chiều sâu của mối quan hệ giữa khách hàng và thương hiệu (Hudson, Roth, Madden, \& Hudson, 2015; Smith, Coyle, Lightfoot, \& Scott, 2007). Khách hàng có thể phát triển mối quan hệ tích cực với các thương hiệu khi chúng tương tác với nhau (Hudson et al., 2015).

Crosby và cộng sự (1990) cho rằng chất lượng mối quan hệ là một cấu trúc bậc cao bao gồm hai thành phần: niềm tin và sự hài lòng. Nghiên cứu của Nyffenegger, Krohmer, Hoyer, và Malaer (2015) lại cho thấy các mối quan hệ giữa khách hàng và thương hiệu là: nhận thức và cảm xúc. Chúng đều ảnh hưởng đến lòng trung thành của thương hiệu.

Mô hình thích hợp để đo lườ ng ảnh hưở ng $\mathrm{c}$ ủa các thành phần nhận thức và cảm xúc của các mối quan hệ thương hiệu đến đặc tính thương hiệu là mô hình cam kết thương hiệu (Giovanis \& Athanasopoulou, 2017; Veloutsou, 2015), bao gồm các thành phần: niềm tin, sự hài lòng, cam kết.

Niềm tin: được định nghĩa là "sụ sắn lòng dụa vào một đối tác trao đổi mà người ta tin tương" (Moorman, Zaltman, \& Deshpande, 1992, p. 315). Niềm tin ở đây là tin rằng thương hiệu sẽ hành động thi ện tâm vì lợi ích tốt nhất của khách hàng dựa trên các mục tiêu và giá trị chung (Chaudhuri \& Holbrook, 2001); do đó khuyến khích các đối tác trao đổi tiếp t ục mối quan hệ cam kết (So, King, Sparks, \& Wang, 2016).

Sụ hài lòng: Sự hài lòng của khách hàng liên quan đến thương hiệu là kết quả của việc đánh giá hiệu quả của thương hiệu so với mong đợi (Aaker, Fournier, \& Brasel, 2004). Anderson, Fornell, và Lehmann (1994), cho rằng "sụ hài lòng là đánh giá tổng thể dụa trên kinh nghiệm lâu dài trong việc mua và tiêu thu một sản phẩm hay dịch vụ".

Cam kết: là mong muốn phát triển mối quan hệ ổn định với một đối tác với niềm tin rằng mối quan hệ lâu dài là điều quan trọng để đảm bảo nỗ lực tối đa để duy trì nó (Morgan \& Hunt, 1994).

\section{Ý định chuyển đổi thương hiệu}

Chuyển đổi thương hiệu là sự chấm dứt mối quan hệ với một thương hiệu nhất định và tiến tới một giải pháp hấp dẫn hơn (Ping, 1993). Chuyển đổi thương hiệu có nghĩa là ngườ dùng tù̀ bỏ một nhà cung cấp dịch vụ, sản phẩm (Keaveney \& Parthasarathy, 2001; RundleThiele \& Bennett, 2001). Chuyển đổi từ thương hi ệu này sang thương hiệu khác là một vấn đề quan trọng đối với một doanh nghiệp vì nó cho thấy thương hiệu gốc không còn đáp ứng được mong đợi của khách hàng và thương hiệu mới sẽ hấp dẫn hơn.

\section{Mối quan hệ giữa chất lượng mối quan hệ thương hiệu - khách hàng và ý định chuyển đổi thương hiệu}

Khi khách hàng tin tưởng vào nhà cung cấp dịch vụ, họ sẽ liên tục sử dụng dịch vụ và sẽ giới thiệu nhà cung cấp dịch vụ cho khách hàng tiềm năng (Nyffenegger et al., 2015). Trong điều kiện có chất lượng mối quan hệ thương hiệu cao, khách hàng sẽ thể hiện mức độ hài lòng, cam kết, niềm tin, gắn bó và tình cảm thân tình đối với thương hiệu. Khách hàng cũng có 
khuynh hướng tiến hành các hành vi tích cực, như tự nguyện giúp đỡ tổ chức dịch vụ và các khách hàng khác của tổ chức (Xie, Poon, \& Zhang, 2017). Khi người tiêu dùng không hài lòng với một thương hiệu, cơ hội của người tiêu dùng chuyển sang một lựa chọn hấp dẫn hơn, trở nên lớn hơn (Gulamali \& Persson, 2017). Như vậy, chất lượng mối quan hệ thương hiệu - khách hàng càng mạnh, niềm tin, sự hài lòng và cam kết của khách hàng với thương hiệu càng cao thì ý định chuyển đổi thương hiệu của khách hàng càng thấp. Dựa trên những lập luận trên có giả thuyết $\mathrm{H} 1$ như sau:

H1: Chất lượng mối quan hệ thuơng hiệu - khách hàng tác động ngược chiều lên ý định chuyển đổi thương hiệu

\section{Mối quan hệ giữa trách nhiệm xã hội của doanh nghiệp và ý định chuyển đổi} thương hiệu, chất lượng mối quan hệ thương hiệu - khách hàng

Khách hàng cảm thấy tự tin về chất lượng sản phẩm hoặc dịch vụ khi họ tin cậy doanh nghiệp và tin tưởng rằng các doanh nghiệp đáng tin cậy sẽ có trách nhiệm đạo đức và xã hội lớn hơn. Stanaland, Lwin, và Murphy (2011) cho rằng CSR khuyến khích nguời tiêu dùng tin tưởng thương hiệu.

Khách hàng còn có thể sẽ hài lòng hơn nếu các nhà cung cấp sản phẩm có trách nhiệm xã hội cao hơn. CSR có thể làm tăng ích lợi và giá trị được nhận thức, từ đó tăng cường sự hài lòng của khách hàng. McDonald và Lai (2011) và Pérez và del Bosque (2015) tìm thấy mối quan hệ tích cực đáng kể giữa CSR và sự hài lòng của khách hàng.

Bên cạnh đó, CSR còn tác động đến sự cam kết của khách hàng (Inoue, Funk, \& McDonald, 2017). Khi khách hàng tin cậy thông qua trách nhiệm xã hội của doanh nghiệp, họ có xu hướng gắn bó với thương hiệu hơn, giảm ý định chuyển đổi thương hiệu và chất lượng mối quan hệ thương hiệu - khách hàng càng được củng cố hơn. Từ những phân tích trên có các giả thuyết $\mathrm{H} 2, \mathrm{H} 3$ như sau:

H2: Trách nhiệm xã hội có tác động ngự̛c chiều lên ý định chuyển đổi thương hiệu

H3: Trách nhiệm xã hội của doanh nghiệp tác động cùng chiều lên chất lượng mối quan hệ thương hiệu - khách hàng

\section{Mô hình nghiên cứu:}

Trên cơ sở phân tích lý thuyết, mô hình nghiên cứu được đề xuất như Hình 2 sau. 


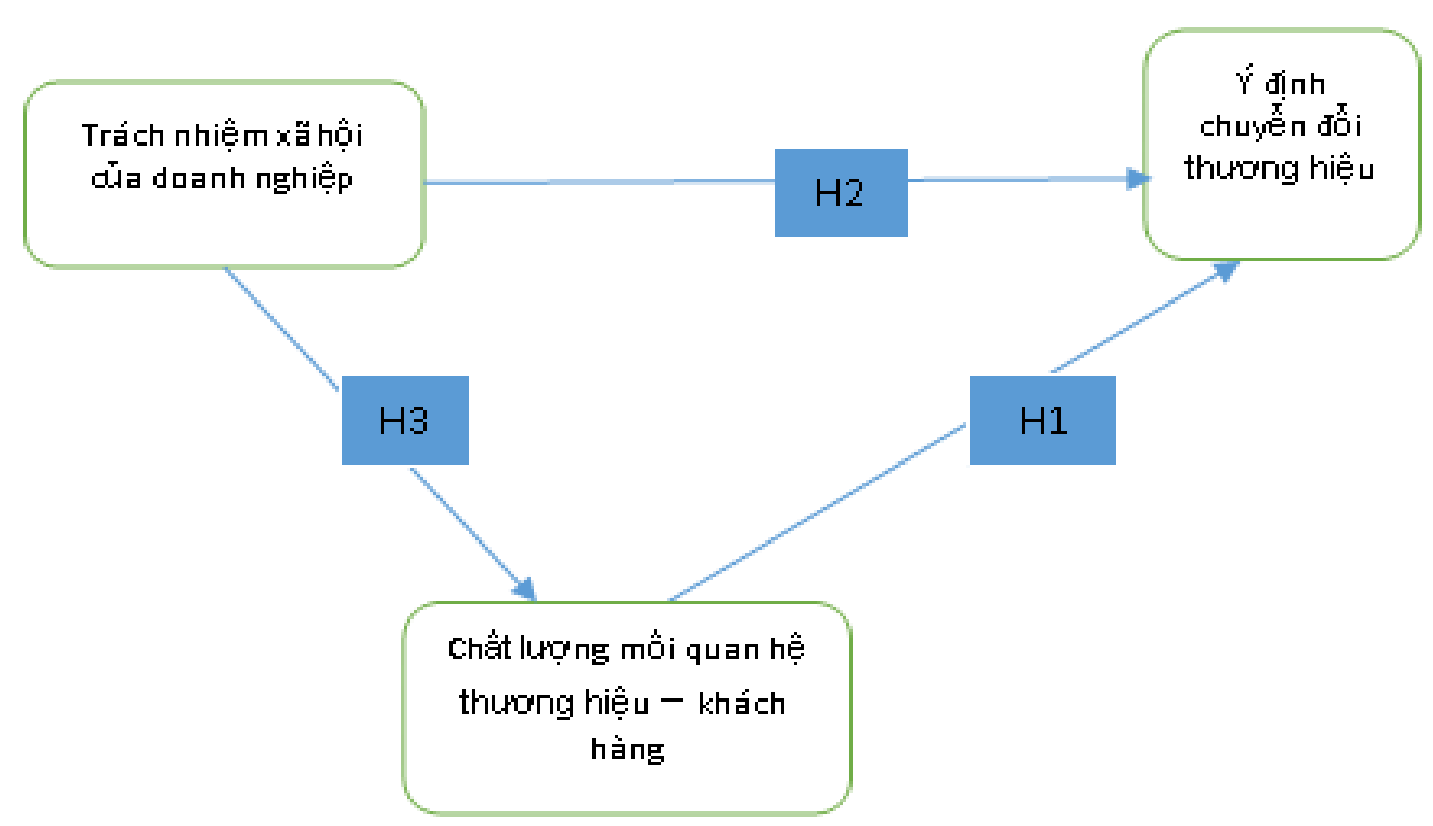

Hình 2. Mô hình nghiên cứu đề xuất

\section{Dữ liệu và phương pháp nghiên cứu}

\subsection{Dũ liệu ngiên cứu}

Nghiên cứu chính thức được thực hiện bằng phương pháp nghiên cứu định lượng, thu thập dữ liệu bằ ng cách phỏng vấ $n$ khách hàng trực tiếp thông qua bảng câu hỏi chi tiết; được thực hiện ở Thành phố Hồ Chí Minh vào từ ngày 01/10/2017 - 30/10/2017. Đối tượng khảo sát là những khách hàng mua sắm ở siêu thị. Tổng số phiế u điề u tra là 530 phiếu; tổng số phiếu thu về: 521; số phiếu không đạt yêu cầu do không điền đầy đủ và bỏ sót câu hỏi là: 14; số phiếu hợp lệ là: 507. Thang đo Likert 7 bậc (từ 1 : Hoàn toàn không đồng ý đến 7 : hoàn toàn đồng ý). Mẫu được chọn theo phương pháp thuận tiện. Số lượng mẫu được lấy theo nguyên tắc 5:1 (Nguyen, 2011).

\section{Thang do}

Thang đo các khái niệm trong mô hình nghiên cứu được phát triển dựa trên các thang đo gốc của các nghiên cứu trước đây và được điều chỉnh sau nghiên cứu định tính. Tất cả các thang đo trong mô hình nghiên cứu đều là thang đo đa biến. Mô hình có 7 khái niệm nghiên cứu với 34 biến quan sát được trình bày trong Bảng 1 . 


\section{Bảng 1}

Thang đo các khái niệm trong mô hình và nguồn gốc

\begin{tabular}{|c|c|c|}
\hline Khái niệm nghiên cứu & Số BQS & Nguồn gốc \\
\hline $\begin{array}{l}\text { Trách nhiệm kinh tế } \\
\text { 1)Thương hiệu X cố gắng tối đa hóa lợi nhuận để duy trì } \\
\text { hoạt động kinh doanh; 2) thương hiệu X cố găng xây dựng } \\
\text { mối quan hệ vững chắc với khách hàng để đảm bảo sự thành } \\
\text { công kinh tế lâu dài; } 3 \text { ) thương hiệu X cố gắng không ngừng } \\
\text { nâng cao chất lượng dịch vụ mà họ cung cấp; 4) thương hiệu } \\
X \text { cố gắng để có một chính sách giá cả tốt nhất cho khách } \\
\text { hàng; 5) thương hiệu X cố gắng hết sức để làm việc hiệu } \\
\text { quả hơn; } 6 \text { ) thương hiệu X cố gắng tổ chức dịch vụ đi kèm: } \\
\text { an ninh, giữ xe, phòng cháy chữa cháy, vệ sinh đáp ứng nhu } \\
\text { cầu của khách hàng. }\end{array}$ & 06 & $\begin{array}{l}\text { Alvarado và cộng } \\
\text { sự (2017) } \\
\text { Nghiên cứu định } \\
\text { tính (thảo luận } \\
\text { nhóm) }\end{array}$ \\
\hline 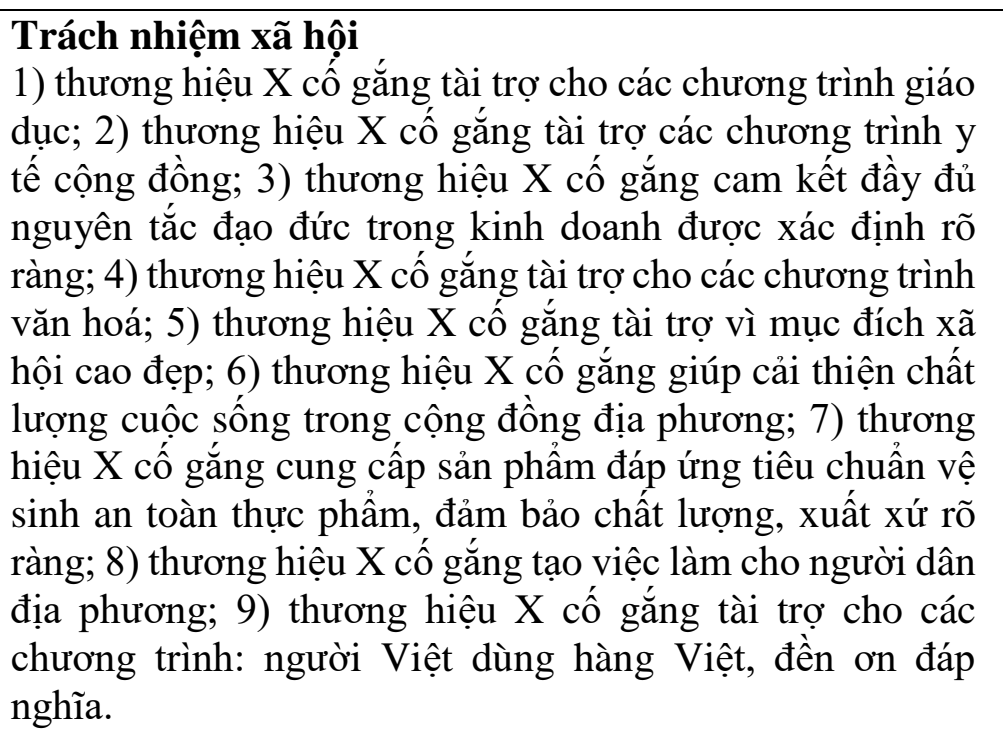 & 09 & $\begin{array}{l}\text { Alvarado và } \\
\text { cộng sự (2017) } \\
\text { Nghiên cứu định } \\
\text { tính (thảo luận } \\
\text { nhóm) }\end{array}$ \\
\hline $\begin{array}{l}\text { Trách nhiệm môi trường } \\
\text { 1) thương hiệu X cố gắng tài trợ các chương trình thân } \\
\text { thiện với môi trường; 2) thương hiệu X cố gắng phân bồ } \\
\text { các nguồn lực để cung cấp các dịch vụ thân thiện với môi } \\
\text { trường; } 3 \text { ) thương hiệu X cố gắng thực hiện các chương } \\
\text { trình để giảm ô nhiếm; 4) thương hiệu } X \text { cố gắng bảo vệ } \\
\text { môi trường; 5) thương hiệu X cồ gắng chỉ sử dụng các } \\
\text { nguồn tài nguyên thiên nhiên cần thiết. }\end{array}$ & 05 & $\begin{array}{l}\text { Alvarado và } \\
\text { cộng sự (2017) } \\
\text { Nghiên cứu định } \\
\text { tính (thảo luận } \\
\text { nhóm) }\end{array}$ \\
\hline $\begin{array}{l}\text { Niềm tin } \\
\text { 1) Tôi tin tưởng thương hiệu } X ; 2) X \text { là một thương } \\
\text { hiệu trung thực; } 3 \text { ) X là một thương hiệu an toàn; } \\
\text { 4) } X \text { hành động vì lợi ích tốt nhất của khách hàng; } \\
\text { 5) X nỗ lực hiếu khách hàng }\end{array}$ & 05 & $\begin{array}{l}\text { Nyffenegger và } \\
\text { cộng sự (2015); } \\
\text { Lo và Im (2014). } \\
\text { Nghiên cứu định } \\
\text { tính (thảo luận }\end{array}$ \\
\hline
\end{tabular}




\begin{tabular}{|c|c|c|}
\hline Khái niệm nghiên cứu & Số BQS & Nguồn gốc \\
\hline $\begin{array}{l}\text { Sự hài lòng } \\
\text { 1) Tôi luôn hài lòng với quyết định mua hàng của mình } \\
\text { với thương hiệu X; 2) Tôi hoàn toàn hài lòng với thương } \\
\text { hiệu X; 3) Thương hiệu X cung cấp chính xác những gì } \\
\text { tôi mong đợi từ một siêu thị; 4) Thương hiệu X luôn tạo } \\
\text { thiện cảm với khách hàng: tặng quà sinh nhật, quà tết, } \\
\text { chương trình dành cho khách hàng thân thiêt... }\end{array}$ & 04 & $\begin{array}{l}\text { Nyffenegger và } \\
\text { cộng sự (2015) } \\
\text { Nghiên cứu định } \\
\text { tính (thảo luận } \\
\text { nhóm) }\end{array}$ \\
\hline $\begin{array}{l}\text { Cam kết } \\
\text { Tôi cảm thấy gắn bó với thương hiệu X; 2) Tôi cam kết có } \\
\text { mối quan hệ lâu dài với thương hiệu X }\end{array}$ & 02 & $\begin{array}{l}\text { Lo và Im (2014). } \\
\text { Nghiên cứu định } \\
\text { tính (thảo luận } \\
\text { nhóm) }\end{array}$ \\
\hline $\begin{array}{l}\text { Ý định chuyển đổi thương hiệu } \\
\text { 1) Tôi dự định chuyển sang thương hiệu siêu thị khác; } 2 \text { ) } \\
\text { Trong tương lai, tôi sẽ mua sắm ở thương hiệu siêu thị } \\
\text { khác; 3) Tôi sẽ không tiếp tục mua sắm tại thương hiệu } \\
\text { siêu thị X }\end{array}$ & 03 & $\begin{array}{l}\text { Shin và } \\
(2008)\end{array}$ \\
\hline
\end{tabular}

Nguồn: Tổng hợp từ lý thuyết và thảo luận nhóm

\subsection{Phưong pháp nghiên cúu}

Nghiên cứu được thực hiện qua hai bước: nghiên cứu sơ bộ và nghiên cứu chính thức.

Nghiên cứu sơ bộ được thực hiệ $n$ bằng phương pháp nghiên cứu định tính và nghiên cứu định lượng.

Nghiên cưu sơ bộ định tính: Tiến hành thảo luận nhóm tậ p trung với 02 nhóm khách hàng thường mua sắm tại các siêu thị bán lẻ tổng hợp ở Thành phố Hồ Chí Minh (ít nhất một tháng/01 lần). Nhóm thứ nhất có độ tuổi từ 21-30; nhóm thứ 2 có độ tuổi từ 31-45. Thông qua các thang đo của các nghiên cứu trước, đáp viên sẽ được hỏi về tính rõ nghĩa của câu hỏi và tự chọn các bi ến quan sát phù hợp với mình. Các biến quan sát của một thang đo sẽ được chọn theo nguyên tắc các biến quan sát có nhiều người chọn nhất. Các biến quan sát không có người chọn hoặc có ít người chọn sẽ không được đưa vào thang đo. Đồng thời các đáp viên sẻ bổ sung các biến quan sát để đo lường các khái niệm nghiên cứu. Kết quả phỏng vấn được ghi nhận, phát triển và điều chỉnh thành thang đo nháp. Nghiên cứu này được thực hiện trong tháng 09 năm 2017.

Nghiên cưu so bộ định lượ ng: Thang đo nháp được dùng để phỏng vấn thử với mẫu 115 khách hàng theo phương pháp lấy mẫu thuận tiện để kiểm định độ tin cậy của thang đo. Sau bước này, thang đo được hoàn chỉnh và sử dụng cho bước nghiên cứu định lượng chính thức.

Nghiên cưu chính thức được thực hiện bằng phương pháp nghiên cứu định lượng, thu thập dữ liệu bằng cách phỏng vấn khách hàng trực tiếp thông qua bảng câu hỏi chi tiết tại Thành phố Hồ Chí Minh vào tháng 10 năm 2017. Kích thước mẫu của nghiên cứu này là 507 . Mục tiêu của nghiên cứu này là khẳng định lại độ tin cậy, giá trị của các thang đo đã thiết kế và điều chỉnh cho phù hợp với thị trườ ng Việt Nam; kiểm định mô hình lý thuyết và các giả thuyết. 
Phương pháp phân tích cấu trúc tuyến tính SEM (Structural Equation Modeling) được sử dụng để kiểm định mô hình lý thuyết và các giả thuyết.

\section{Kết quả và thảo luận}

\subsection{Kết quả nghiên cúu}

Đặc điểm mẫu thống kê: Mẫu thống kê gồm 396 nữ chiếm 78,1\%; 111 nam chiếm 21,9\%. Số người có độ tuổi từ 25-34 chiếm đa số, 205 người chiếm 40,4\%; ở độ tuổi từ 35-44 có 105 người, chiếm 20,7\%. Trình độ học vấn, phần lớn có trình độ cao đẳng - đại học 476 người (chiếm 93,9\%). Xét về thu nhập, mức thu nhập phổ biến là 10-20 triệu/tháng 181 người chiếm $37,5 \%$; kế đến là từ 5-10 triệu/tháng có 127 người chiếm $25 \%$. Kết quả kiểm định thang đo được trình bày trong Bảng 2 .

\section{Bảng 2}

Bảng tóm tắt kết quả kiểm định thang đo

\begin{tabular}{|c|c|c|c|c|c|c|}
\hline \multirow[b]{2}{*}{ Thang đo } & \multirow[b]{2}{*}{ Thành phần } & \multirow{2}{*}{$\begin{array}{c}\text { Số } \\
\text { BQS }\end{array}$} & \multicolumn{2}{|c|}{ Độ tin cậy } & \multirow{2}{*}{$\begin{array}{c}\text { Phưo'ng } \\
\text { sai } \\
\text { trích }\end{array}$} & \multirow{2}{*}{$\begin{array}{c}\text { Tải } \\
\text { nhân } \\
\text { tố bình } \\
\text { quân }\end{array}$} \\
\hline & & & $\begin{array}{l}\text { Cronbach } \\
\text { alpha }\end{array}$ & Tổng họ̣p & & \\
\hline \multirow{3}{*}{$\begin{array}{l}\text { Trách nhiệm } \\
\text { xã hội của } \\
\text { doanh nghiệp }\end{array}$} & $\begin{array}{l}\text { Trách nhiệm } \\
\text { kinh tế }\end{array}$ & 4 & 0.806 & 0.8144 & 0.5232 & 0.7233 \\
\hline & $\begin{array}{l}\text { Trách nhiệm } \\
\text { xã hội }\end{array}$ & 7 & 0.882 & 0.8902 & 0.5369 & 0.7324 \\
\hline & $\begin{array}{l}\text { Trách nhiệm } \\
\text { môi trường }\end{array}$ & 5 & 0.901 & 0.8446 & 0.5211 & 0.7216 \\
\hline \multirow{3}{*}{$\begin{array}{l}\text { Chất lượng } \\
\text { mối quan hệ } \\
\text { thương hiệu - } \\
\text { khách hàng }\end{array}$} & Niềm tin & 3 & 0.867 & 0.8960 & 0.6334 & 0.7950 \\
\hline & Sự hài lòng & 4 & 0.929 & 0.8973 & 0.6861 & 0.8280 \\
\hline & Cam kết & 2 & 0.865 & 0.8821 & 0.7892 & 0.8880 \\
\hline \multicolumn{2}{|c|}{ Chuyển đổi thương hiệu } & 3 & 0.903 & 0.8310 & 0.6211 & 0.7880 \\
\hline
\end{tabular}

Nguồn: Kết quả xử lý từ dữ liệu điều tra

Kết quả kiểm định SEM cho thấy mô hình có 397 bậc tự do, chi -square $=569.708$ ( $\mathrm{p}=0.000)$, Chi-square/df $=1.435<3$. Các chỉ số: $\mathrm{TLI}=0.967 ; \mathrm{CFI}=0.978 ; \mathrm{GFI}=0.933$ đều lớn hơn 0.9 và $\mathrm{RMSEA}=0.029<0.08$. Vì vậy, có thể kết luậ $\mathrm{n}$ mô hình phù hợp với dữ liệu thị trường (xem Hình 3 ). 


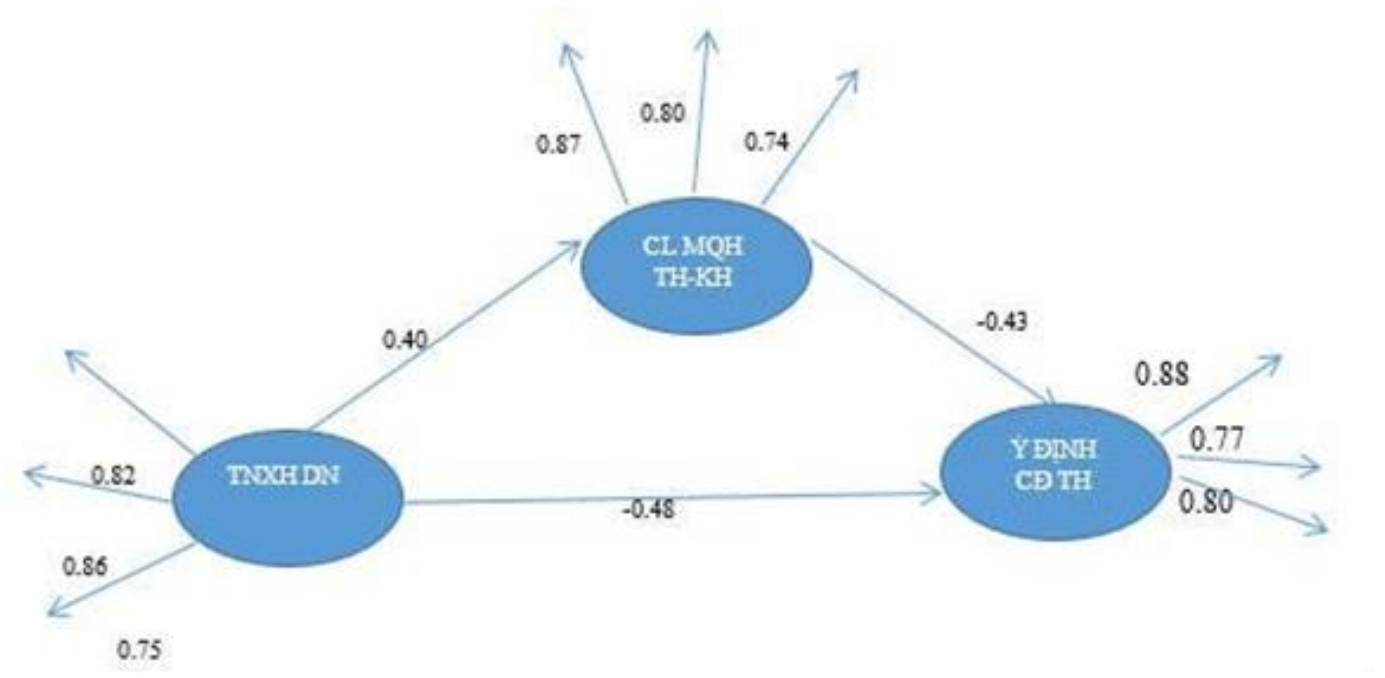

Hình 3. Kết quả SEM (chuẩn hóa) của mô hình lý thuyết

Nguồn: Kết quả xử lý dữ liệu điều tra

Kết quả ước lượng (chuẩn hóa) của các tham số trong Bảng 3 cho thấy, trách nhiệm xã hội của doanh nghiệp có tác động trực tiếp và thuận chiều lên chất lượng mối quan hệ thương hiệu - khách hàng; tác động trực tiếp và ngược chiều lên ý định chuyển đổi thương hiệu. Chất lượng mối quan hệ thương hiệu - khách hàng có tác động trực tiếp và nghịch chiều lên ý đị nh chuyể $\mathrm{n}$ đổi thương hiệu. Những tác động được ghi nhận trên đây đều phù hợp vớ i giả thuyết đề ra và đều có ý ngh ĩa thống kê vì có $\mathrm{p}<0.05$. Như vậy, các giả thuyết $\mathrm{H} 1, \mathrm{H} 2, \mathrm{H} 3$ đều được chấp nhận.

\section{Bảng 3}

Hệ số hồi quy chưa chuẩn hóa của mô hình lý thuyết

\begin{tabular}{|l|l|l|c|c|c|c|}
\hline \multicolumn{2}{|c|}{ Tương quan } & $\begin{array}{c}\text { Ước } \\
\text { lượng }\end{array}$ & SE & CR & P-value \\
\hline CSR & $->$ & $\begin{array}{l}\text { Chất lượng mối quan } \\
\text { hệ thương hiệu - } \\
\text { khách hàng }\end{array}$ & 0.341 & 0.052 & 6.615 & $* * *$ \\
\hline CSR & $->$ & $\begin{array}{l}\text { Ý định chuyển đổi } \\
\text { thương hiệu }\end{array}$ & -0.571 & 0.063 & -9.104 & $* * *$ \\
\hline $\begin{array}{l}\text { Chất lượng mối } \\
\text { quan hệ thương } \\
\text { hiệu - khách hàng }\end{array}$ & $->$ & $\begin{array}{l}\text { Ý định chuyển đổi } \\
\text { thương hiệu }\end{array}$ & -0.612 & 0.084 & -7.299 & $* * *$ \\
\hline
\end{tabular}

Nguồn: Kết quả xử lý từ dữ liệu điều tra

Bảng 4 cho thấy tác động trực tiếp, gián tiếp và tác động tổng hợp của các biến độc lập lên các biến phụ thuộc trong mô hình lý thuyết.

Kết quả tính toán ở Bảng 4 cho thấy: CSR có tác động trực tiếp, cùng chiều và khá mạnh đến ch ất lượng mối quan hệ thương hiệu - khách hàng $(\beta=0.34)$; tác động trực tiếp và ngược chiề u và khá mạnh lên ý định chuyển đổi thương hiệu $(\beta=-0.57)$. CSR tác động gián tiếp lên 
ý định chuyển đổi thương hiệu thông qua chất lượng mối quan hệ thươ ng hiệu - khách hàng với $\beta=-0.20$, tổng tác động $\beta$ tổng $=-0.77$. Bên cạnh đó, chất lượng mối quan hệ thương hiệu khách hàng tác động trực tiếp và ngược chiều lên ý định chuyển đổi thương hiệu $(\beta=-0.612)$. Điề u này cho thấy tầm quan trọng đặc biệt của biến trung gian mối quan hệ thương hiệu - khách hàng đến ý định chuyển đổi thương hiệu của khách hàng.

\section{Bảng 4}

Kết quả tác động trực tiếp, gián tiếp và tổng hợp giữa các khái niệm trong mô hình nghiên cứu

\begin{tabular}{|c|c|c|c|}
\hline & Tác động & CSR & BRQ \\
\hline \multirow{3}{*}{ BRQ } & Trực tiếp & 0.34 & \\
\cline { 2 - 4 } & Gián tiếp & 0 & \\
\cline { 2 - 4 } & Tổng & 0.34 & -0.61 \\
\hline \multirow{3}{*}{ Sw } & Trực tiếp & -0.57 & 0 \\
\cline { 2 - 4 } & Gián tiếp & -0.20 & -0.61 \\
\cline { 2 - 4 } & Tổng & -0.77 & \\
\hline
\end{tabular}

Nguồn: Kết quả xử lý tử dữ liệu điều tra

\subsection{Thảo luận kết quả nghiên cúu}

Nghiên cứu này nhằm mục đích giải thích rõ hơn mối quan hệ giữa $\mathrm{CSR}$, chất lượng mối quan hệ thương hiệu - khách hàng và ý định chuyển đổi thương hiệu.

Về mối quan hệ giữa các khái niệm, CSR tác động trực tiếp cùng chiều khá mạnh đến chất lượng mối quan hệ thương hiệu - khách hàng $(\beta=0.34)$. Có nghĩa, khi khách hàng cảm nhận các hoạt động CSR thì sẽ tang cường chất lượng mối quan hệ thương hiệu - khách hàng. Kết quả này phù hợp với nghiên cứu của Lev và cộng sự (2010); Hur và cộng sự (2016); Nyadzayo và Khajehzadeh (2016).

CSR có tác động trực tiếp ngược chiều khá mạnh lên ý đị nh chuyển đổi thương hiệu ( $\beta$ $=-0.57)$ và tác động gián tiếp ở mức thấp hơn $(\beta=-0.20)$ thông qua chất lượng mối quan hệ thương hiệu - khách hàng. Điều này phù hợp với các nghiên cứu trước đây khi cho rằng $\mathrm{CSR}$ có ảnh hưởng trực tiếp và gián tiếp đến hành vi của khách hàng (Abdeen et al., 2016; Öberseder et al., 2011). Kết quả đã chứng minh tầm quan trọng của vai trò trung gian của chất lượng mối quan hệ thương hiệu - khách hàng trong mối quan hệ giữa CSR và ý định chuyển đổi thương hiệu. Đây là kết quả mới của nghiên cứu.

\section{Kết luận và gọi ý chính sách}

\subsection{Kết luận}

Dựa vào các nghiên cứu trước đây, nhóm tác gi ả đã xây dựng mô hình mối quan hệ giữa CSR, chất lượ ng mối quan hệ thương hiệu - khách hàng và ý định chuyển đổi thương hiệu. Kết quả cho thấy thang đo ý định chuyển đổi thương hiệu đạt độ tin cậy cao. CSR tác động trực tiếp, cùng chiều đến ý định chuyển đổi thương hiệu. Kết quả nghiên cứu này hoàn toàn phù hợp với các nghiên cứu trước đó khi cho rằng nhận thức về CSR ảnh hưởng đến ý định hành vi của khách hàng và các hành vi thực tế (Abdeen et al., 2016; Berens, van Riel, \& van Rekom, 2007; Öberseder et al., 2011). 
Đồng thời nghiên cứu này cũng cho thấy chất lượng mối quan hệ thương hiệu - khách hàng có vai trò trung gian trong mối quan hệ giữa $\mathrm{CSR}$ và ý định chuyển đổi thương hiệu; phù hợp với kết quả nghiên cứu của Luo và Bhattacharya (2006); Carroll và Shabana (2010) cho rằng hiệu quả của CSR đối với hành vi của khách hàng chủ yếu phụ thuộc vào các biến trung gian.

\section{Hạn chế và hướng nghiên cứu tiếp theo}

Mặc dù nghiên cứu đã nỗ lực chọn mẫu nhân khẩu học khác nhau để nâng cao tính đại diện; tuy nhiên mẫu chỉ được thu thập tại Thành phố Hồ Chí Minh, Việt Nam. Nghiên cứu tiếp theo có thể mở rộng phạm vi khảo sát để có được thông tin tốt hơn. Đồng thời, mở rộng nghiên cứu sang các lĩnh vực dịch vụ khác để kiểm định lại thang đo và mô hình.

\subsection{Gọi ý chính sách và kiến nghị giải pháp}

Nghiên cứu này dựa trên lý thuyết bộ ba phát triển bền vững TBL cho rằng CSR bao gồm: trách nhiệm kinh tế, trách nhiệm xã hội, trách nhiệm môi trường và ứng dụng trong lĩnh vực dịch vụ tại Việt Nam. Kết quả chỉ ra rằng ba thành phần này ảnh hưởng tích cực đến CSR. Vì vậy, phải tập trung vào cả ba trách nhiệm này để nâng cao cảm nhận của khách hàng về CSR. Trong ba thành phần của CSR thì thành phần trách nhiệm xã hội được khách hàng cảm nhận rõ ràng nhất, vì vậy cần đặc biệt chú trọng đến các hoạt động này. Đặc biệt, trong bối cảnh ngành dịch vụ tại các siêu thị bán lẻ tại Việt Nam thì cần quan tâm thêm đến việc thể hiện trách nhiệm xã hội thông qua cung cấp sản phẩm đáp ứng tiêu chuẩn vệ sinh an toàn thực phẩm, đảm bảo chất lượng, xuất xứ rõ ràng; tạo việc làm cho người dân địa phương.

Đồng thời, để góp phần nâng cao chất lượ ng mối quan hệ thương hiệu khách hàng, với đặc thù văn hóa của Việt Nam; các thương hiệu cần chú trọng đến việc tạo thiện cảm với khách hàng thông qua các hoạt động như: tặng quà sinh nhật, quà tết, chương trình dành cho khách hàng thân thiết, ...

Khi khách hàng cảm nhận tốt về hoạt động CSR thì chất lượng mối quan hệ thương hiệu - khách hàng càng được nâng cao và làm giảm ý định chuyển đổi thương hiệu của khách hàng. CSR cũng tác động trực tiếp, cùng chiều lên ý định chuyển đổi thương hiệu của khách hàng. Vì vậy, muốn phát triển thương hiệu cần phải dành ngân sách, nguồn lực để hỗ trợ các hoạt động CSR.

\section{Tài liệu tham khảo}

Aaker, J., Fournier, S., \& Brasel, S. A. (2004). When good brands do bad. Journal of Consumer Research, 31(1), 1-16.

Abdeen, A., Rajah, E., \& Gaur, S. S. (2016). Consumers' beliefs about firm's CSR initiatives and their purchase behaviour. Marketing Intelligence \& Planning, 34(1), 2-18.

Alvarado-Herrera, A., Bigne, E., Aldas-Manzano, J., \& Curras-Perez, R. (2017). A scale for measuring consumer perceptions of corporate social responsibility following the sustainable development paradigm. Journal of Business Ethics, 140(2), 243-262. 
Anderson, E. W., Fornell, C., \& Lehmann, D. R. (1994). Customer satisfaction, market share, and profitability: Findings from Sweden. The Journal of Marketing, 58(3), 53-66.

Berens, G., van Riel, C. B. M., \& van Rekom, J. (2007). The CSR-Quality trade-off: When can corporate social responsibility and corporate ability compensate each other? Journal of Business Ethics, 74(3), 233-252. doi:10.1007/s10551-006-9232-0

Bhattacharya, C. B., Rao, H., \& Glyn, M. A. (1995). Understanding the bond of identification: An investigation of its correlates among art museum members. Journal of Marketing, 59(4), 46-57.

Bhattacharya, C. B., \& Sen, S. (2003). Consumer-company identification: A framework for understanding consumers' relationships with companies. Journal of Marketing, 67(2), 76-88.

Carroll, A. B., \& Shabana, K. M. (2010). The business case for corporate social responsibility: A review of concepts, research and practice. International Journal of Management Reviews, 12(1), 85-105.

Chaudhuri, A., \& Holbrook, M. B. (2001). The chain of effects from brand trust and brand affect to brand performance: The role of brand loyalty. Journal of Marketing, 65(2), 8193.

Crosby, L. A., Evans, K. R., \& Cowles, D. (1990). Relationship quality in services selling: An interpersonal influence perspective. The Journal of Marketing, 54(3), 68-81.

Du, S., Bhattacharya, C. B., \& Sen, S. (2011). Corporate social responsibility and competitive advantage: Overcoming the trust barrier. Management Science, 57(9), 1528-1545.

Elkington, J. (1997). Cannibals with forks. The triple bottom line of 21st century business. Oxford, UK: Capstone.

Fatma, M., Khan, I., \& Rahman, Z. (2016). How does corporate association influence consumer brand loyalty? Mediating role of brand identification. Journal of Product \& Brand Management, 25(7), 629-641. doi:10.1108/JPBM-07-2015-0932

Fulop, G., Hisrich, R. D., \& Szegedi, K. (2000). Business ethics and social responsibility in transition economies. Journal of Management Development. 19(1), 5-31. doi:10.1108/02621710010308135

Giovanis, A., \& Athanasopoulou, P. (2017). Gen Y-ers' brand loyalty drivers in emerging devices. Marketing Intelligence \& Planning, 35(6), 805-821.

Gulamali, A., \& Persson, J. (2017). The social media influencer and brand switching. Retrieved December 12, 2017, from http://lup.lub.lu.se/student-papers/record/8910352

Hudson, S., Roth, M. S., Madden, T. J., \& Hudson, R. (2015). The effects of social media on emotions, brand relationship quality, and word of mouth: An empirical study of music festival attendees. Tourism Management, 47, 68-76. 
Hur, W. M., Kim, H., \& Jang, J. H. (2016). The role of gender differences in the impact of CSR perceptions on corporate marketing outcomes. Corporate Social Responsibility and Environmental Management, 23(6), 345-357.

Inoue, Y., Funk, D. C., \& McDonald, H. (2017). Predicting behavioral loyalty through corporate social responsibility: The mediating role of involvement and commitment. Journal of Business Research, 75, 46-56.

Kakabadse, N. K., \& Rozuel, C. (2006). Meaning of corporate social responsibility in a local French hospital: A case study. Society and Business Review, 1(1), 77-96.

Kale, P., \& Singh, H. (2007). Building firm capabilities through learning: The role of the alliance learning process in alliance capability and firm-level alliance success. Strategic Management Journal, 28(10), 981-1000.

Keaveney, S. M., \& Parthasarathy, M. (2001). Customer switching behavior in online services: An exploratory study of the role of selected attitudinal, behavioral, and demographic factors. Journal of the Academy of Marketing Science, 29(4), 374-390.

Lacey, R., Kennett-Hensel, P. A., \& Manolis, C. (2015). Is corporate social responsibility a motivator or hygiene factor? Insights into its bivalent nature. Journal of the Academy of Marketing Science, 43(3), 315-332.

Lev, B., Petrovits, C., \& Radhakrishnan, S. (2010). Is doing good good for you? How corporate charitable contributions enhance revenue growth. Strategic Management Journal, 31(2), 182-200.

Lin, C. P., \& Liu, M. L. (2017). Examining the effects of corporate social responsibility and ethical leadership on turnover intention. Personnel Review, 46(3), 526-550.

Lindgreen, A., Swaen, V., \& Johnston, W. J. (2009). Corporate social responsibility: An empirical investigation of US organizations. Journal of Business Ethics, 85, 303-323.

Lo, A. S. Y., \& Im, H. H. (2014). Drivers of customer-brand relationship quality: A case of mainland Chinese hotel loyalty program members. Journal of Travel \& Tourism Marketing, 31(7), 763-782.

Loureiro, S. M., Sardinha, I. M. D., \& Reijnders, L. (2012). The effect of corporate social responsibility on consumer satisfaction and perceived value: The case of the automobile industry sector in Portugal. Journal of Cleaner Production, 37, 172-178.

Luo, X., \& Bhattacharya, C. B. (2006). Corporate social responsibility, customer satisfaction, and market value. Journal of Marketing, 70(4), 1-18.

McDonald, L. M., \& Lai, H. C. (2011). Impact of corporate social responsibility initiatives on Taiwanese banking customers. International Journal of Bank Marketing, 29(1), 50-63.

Moorman, C., Zaltman, G., \& Deshpande, R. (1992). Relationships between providers and users of market research: The dynamics of trust within and between organizations. Journal of Marketing Research, 29(3), 314-328. 
Morgan, R. M., \& Hunt, S. D. (1994). The commitment-trust theory of relationship marketing. Journal of Marketing, 58(3), 20-38.

Nguyen, T. D. (2011). Giáo trình Phuoong pháp nghiên cúu khoa học trong kinh doanh [Curriculum Methods of scientific research in business]. Hanoi, Vietnam: Nhà xuất bản Lao động - Xã hội.

Nyadzayo, M. W., \& Khajehzadeh, S. (2016). The antecedents of customer loyalty: A moderated mediation model of customer relationship management quality and brand image. Journal of Retailing and Consumer Services, 30, 262-270.

Nyffenegger, B., Krohmer, H., Hoyer, W. D., \& Malaer, L. (2015). Service brand relationship quality: Hot or cold? Journal of Service Research, 18(1), 90-106.

Öberseder, M., Schlegelmilch, B. B., \& Gruber, V. (2011). Why don't consumers care about CSR? A qualitative study exploring the role of CSR in consumption decisions. Journal of Business Ethics, 104(4), 449-460.

Öberseder, M., Schlegelmilch, B. B., Murphy, P. E., \& Gruber, V. (2014). Consumers' perceptions of corporate social responsibility: Scale development and validation. Journal of Business Ethics, 124(1), 101-115.

Pérez, A., \& del Bosque, R. I. (2015). Corporate social responsibility and customer loyalty: Exploring the role of identification, satisfaction and type of company. Journal of Services Marketing, 29(1), 15-25.

Ping, R. A. (1993). The effects of satisfaction and structural constraints on retailer exiting, voice, loyalty, opportunism, and neglect. Journal of retailing, 69(3), 320-352.

Rundle-Thiele, S., \& Bennett, R. (2001). A brand for all seasons? A discussion of brand loyalty approaches and their applicability for different markets. Journal of Product \& Brand Management, 10(1), 25-37.

Sheth, J. N., Sethia, N. K., \& Srinivas, S. (2011). Mindful consumption: A customer-centric approach to sustainability. Journal of the Academy of Marketing Science, 39(1), 21-39.

Shin, D. H., \& Kim, W. Y. (2008). Forecasting customer switching intention in mobile service: An exploratory study of predictive factors in mobile number portability. Technological Forecasting and Social Change, 75(6), 854-874.

Smith, A. K., \& Bolton, R. N. (1998). An experimental investigation of customer reactions to service failure and recovery encounters: paradox or peril? Journal of Service Research, $1(1), 65-81$.

Smith, T., Coyle, J. R., Lightfoot, E., \& Scott, A. (2007). Reconsidering models of influence: the relationship between consumer social networks and word-of-mouth effectiveness. Journal of Advertising Research, 47(4), 387-397. doi:10.2501/S0021849907070407

So, K. K. F., King, C., Sparks, B. A., \& Wang, Y. (2016). Enhancing customer relationships with retail service brands: The role of customer engagement. Journal of Service Management, 27(2), 170-193. 
Stanaland, A. J., Lwin, M. O., \& Murphy, P. E. (2011). Consumer perceptions of the antecedents and consequences of corporate social responsibility. Journal of Business Ethics, 102(1), 47-55.

Su, L., Swanson, S. R., \& Chen, X. (2015b). The effects of perceived service quality on repurchase intentions and subjective well-being of Chinese tourists: The mediating role of relationship quality. Tourism Management, 52, 82-95.

Van Marrewijk, M. (2003). Concepts and definitions of CSR and corporate sustainability: Between agency and communion. Journal of Business Ethics, 44(2), 95-105.

Veloutsou, C. (2015). Brand evaluation, satisfaction and trust as predictors of brand loyalty: The mediator-moderator effect of brand relationships. Journal of Consumer Marketing, 32(6), 405-421.

Vlachos, P. A., Tsamakos, A., Vrechopoulos, A. P., \& Avramidis, P. K. (2009). Corporate social responsibility: Attributions, loyalty, and the mediating role of trust. Journal of the Academy of Marketing Science, 37(2), 170-180.

Xie, L., Poon, P., \& Zhang, W. (2017). Brand experience and customer citizenship behavior: The role of brand relationship quality. Journal of Consumer Marketing, 34(3), 268-280. 